\title{
Pseudomonas aeruginosa in the home environment of newly infected cystic
} fibrosis patients

\author{
P. Schelstraete*, S. Van daele*, K. De Boeck", M. Proesmans" ${ }^{\#}$ P. Lebecque \\ J. Leclercq-Foucart ${ }^{+}$, A. Malfroot ${ }^{\S}$, M. Vaneechoutte ${ }^{f}$ and F. De Baets*
}

ABSTRACT: The source of acquisition of Pseudomonas aeruginosa in cystic fibrosis (CF) patients remains unknown. Patient-to-patient transmission has been well documented but the role of the environment as a source of initial infection is as yet unclear.

In the present study, the origin of the first $P$. aeruginosa isolate in CF patients was investigated by comparing the $P$. aeruginosa genotype(s) from newly infected patients with genotypes of $P$. aeruginosa isolates from the home environment and from other patients from the same CF centre.

A total of 50 newly infected patients were studied. $P$. aeruginosa could be cultured from $5.9 \%$ of the environmental samples, corresponding to 18 patients. For nine of these, the genotype of the environmental $\boldsymbol{P}$. aeruginosa isolate was identical to the patient's isolate. In total, $72 \%$ of the environmental $\boldsymbol{P}$. aeruginosa isolates were encountered in the bathroom. Patient-to-patient transmission within the CF centre could not be ruled out for three patients.

In summary, a low prevalence of Pseudomonas aeruginosa was found in the home environment of the newly infected cystic fibrosis patients. The bathroom should be targeted in any preventive cleaning procedures. An environmental source of the new infection could not be ruled out in nine patients.

KEYWORDS: Cystic fibrosis, environment, genotyping, Pseudomonas aeruginosa

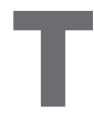
he major pulmonary pathogen in patients with cystic fibrosis (CF) is Pseudomonas aeruginosa. Dehydration of mucus during exacerbations and a defective host defence make CF airways prone to chronic infection with Pseudomonas aeruginosa [1, 2]. By adulthood, $>80 \%$ of patients are infected with this pathogen, which adversely affects lung function and survival $[3,4]$.

To date, the source of initial infection of CF patients with $P$. aeruginosa remains unknown. Possible sources include the environment, person-to-person spread (especially from another person with CF), or through contact with contaminated objects. Patient-to-patient transmission has been well documented. It generally results from prolonged social contact, such as that between siblings [5], close friends or people staying at holiday camps $[6,7]$ and CF rehabilitation centres $[7,8]$.

Epidemiological studies have not confirmed the acquisition of $P$. aeruginosa from the environment. As $P$. aeruginosa is widely present in soil, plants and water $[9,10]$, the environment could be the initial source of infection. The lack of knowledge about the exact role of the environment in the acquisition of $P$. aeruginosa by the CF patient is of concern to parents and physicians, and sometimes leads to questionable preventive measures, such as not drinking tap water unless it has been boiled or not visiting swimming pools [11, 12].

In an attempt to elucidate the source of new strain acquisitions, the current authors carried out a Belgian multicentre study. For all patients who were not initially infected with $P$. aeruginosa, but for whom a recent new infection with $P$. aeruginosa could be documented, cultures of wet surfaces were taken at their homes as soon as possible after detection of the infection. DNA fingerprints of the $P$. aeruginosa isolates from the newly infected patients were compared with fingerprints of $P$. aeruginosa strains recovered from the home environment. Using the Belgian inventory of the DNA fingerprints of most patient $P$. aeruginosa isolates [7], the DNA fingerprints of the $P$. aeruginosa isolates from the newly infected patients were compared with the fingerprints of $P$. aeruginosa isolates from chronically infected CF patients attending the same CF centre as the newly infected patients.

\section{AFFILIATIONS}

*Cystic Fibrosis Centres, Ghent University Hospital, Ghent, \#University Hospital KULeuven, Leuven,

"University Hospital UCL, Louvain-laNeuve,

+University Hospital ULG, Liège §University Hospital UZBrussel, Brussels,

${ }^{f}$ Dept of Clinical Chemistry, Bacteriology and Immunology, Ghent University Hospital, Ghent, Belgium

CORRESPONDENCE

P. Schelstraete

Dept of Paediatrics

Cystic Fibrosis Centre 5K6

Ghent University Hospital

De Pintelaan 185

9000 Gent

Belgium

Fax: 3293323861

E-mail: petra.schelstraete@ugent.be

Received:

July 152007

Accepted after revision:

November 302007

SUPPORT STATEMENT

This study was supported by the Belgian Cystic Fibrosis Association. The present work was previously published as an abstract at the North American Cystic Fibrosis Conference 2006 (Pediatr Pulmonol 2006; 41: Suppl. 29, 325).

STATEMENT OF INTEREST None declared. 


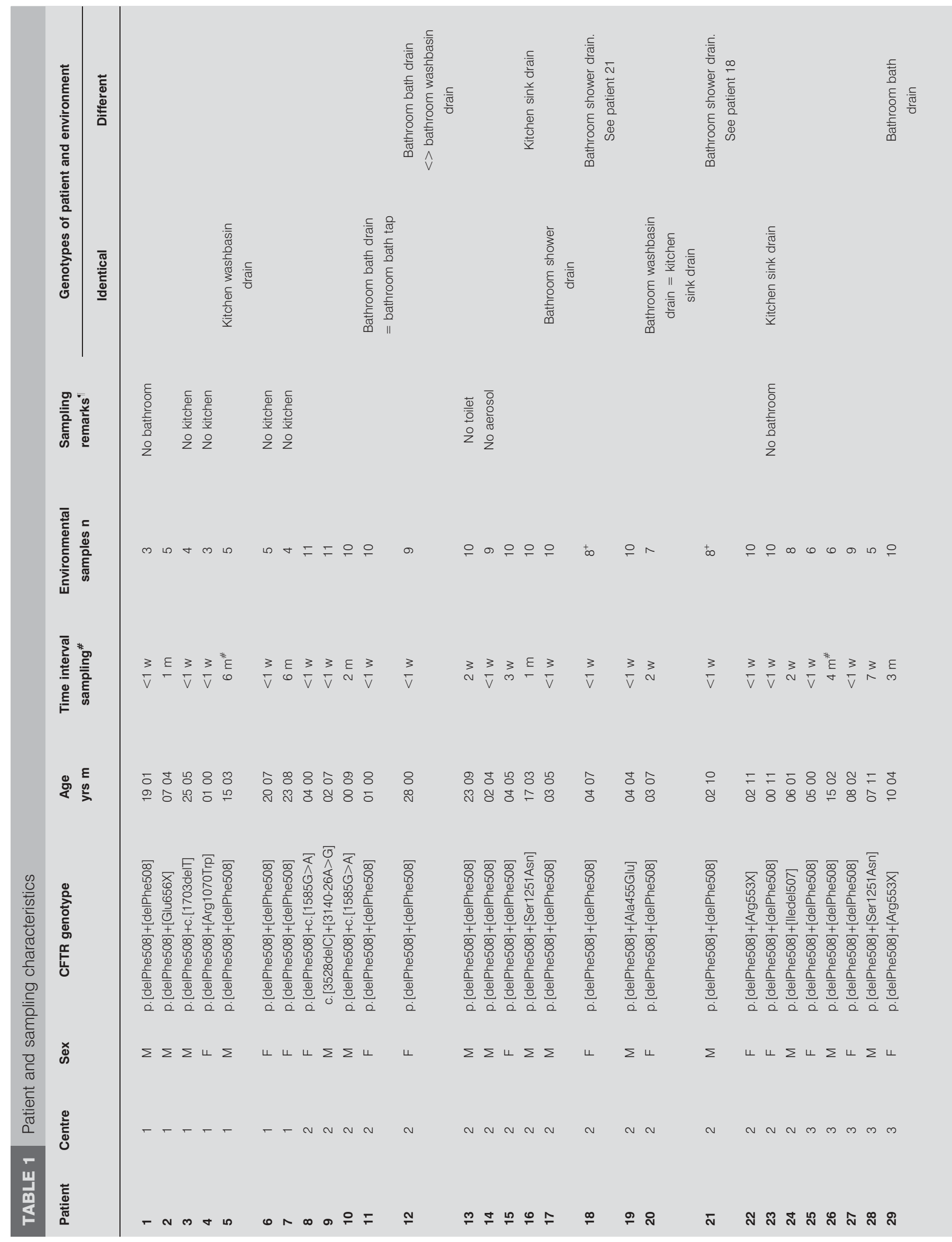




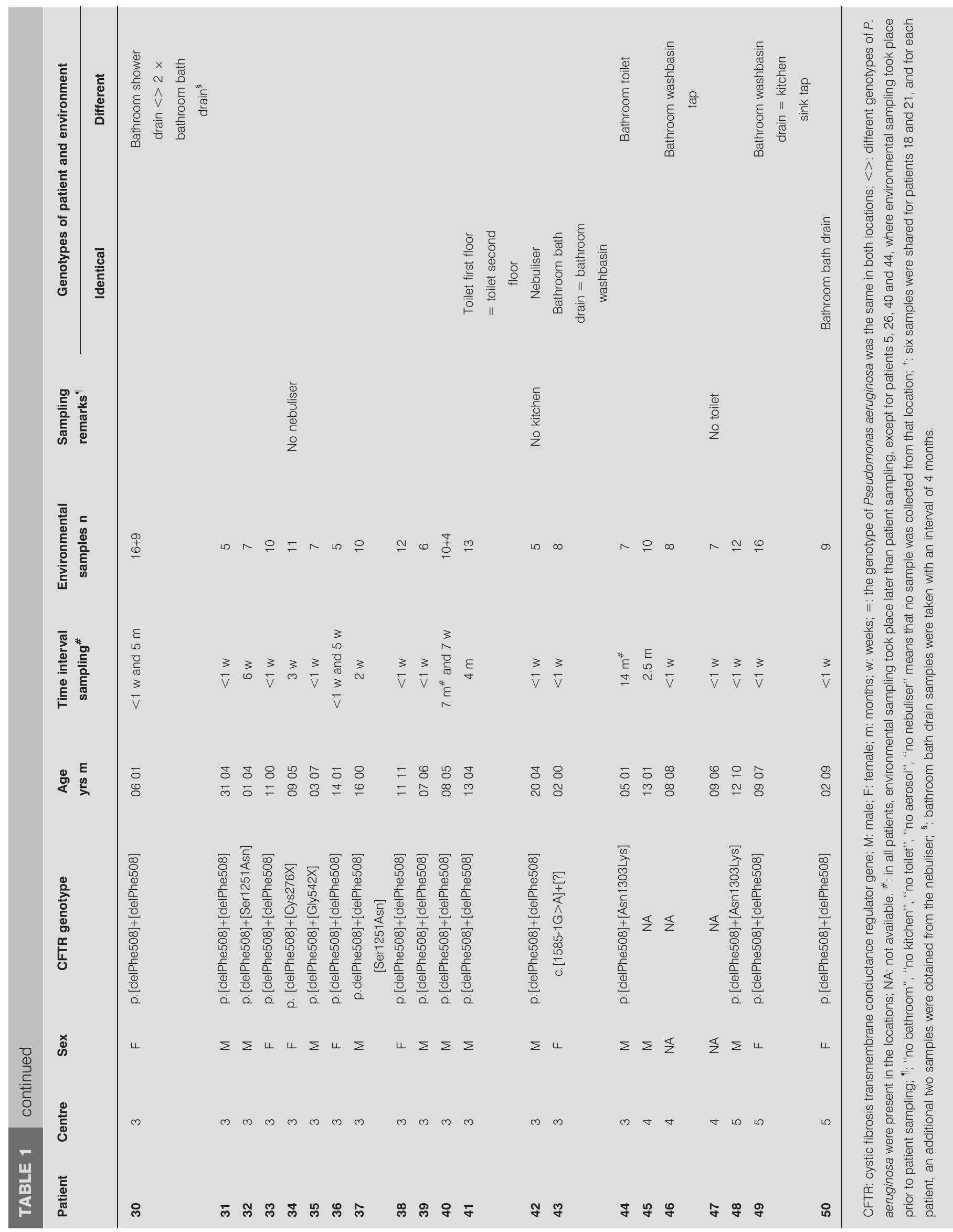




\section{METHODS}

\section{Study population and sampling}

Between January 2003 and December 2005, five Belgian CF centres sent sputum, nasopharyngeal aspirate or an isolate of newly $P$. aeruginosa-infected patients to the microbiology laboratory of Ghent University Hospital (GUH; Ghent, Belgium) for culture, confirmation of the identification and genotyping of the P. aeruginosa isolates. Approval of the Ethics Committee of the GUH was obtained. In each centre, $P$. aeruginosa-negative CF patients were seen at 3-month intervals in segregated consultation rooms or on different consultation days. All newly infected patients were treated for 3 months with oral ciprofloxacin $\left(15 \mathrm{mg} \cdot \mathrm{kg}^{-1}\right.$ b.i.d.) and inhaled colistin $\left(2 \times 10^{6} \mathrm{U}\right.$ b.i.d. $)$ according to the international guidelines [13]. As soon as possible after the diagnosis of a first $P$. aeruginosa infection, the CF centre nurse visited the home of the patient and collected environmental samples. Samples were taken from the nebuliser, the taps and sink in the kitchen, the taps and drains of the bath, the shower and washbasin in the bathroom and the toilet(s). Samples from other wet surfaces, e.g. swimming pools and aquaria, were taken at the discretion of the nurses. A sterile swab (Nuova Aptaca, Canelli, Italy) was used to sample wet surfaces. Surface water $(5 \mathrm{~mL})$ was collected from toilets, swimming pools and aquaria by means of a sterile syringe. The medication container of the nebuliser was rinsed with $5 \mathrm{~mL}$ of sterile saline, which was poured into a sterile sample container.

\section{Microbiology}

Sputum and environmental samples were inoculated onto McConkey agar (BBL Becton Dickinson, Cockeysville, MD, USA). After 2 days of incubation at $37^{\circ} \mathrm{C}$, morphologically different lactose-negative colonies were picked, subcultured on $5 \%$ sheep blood agar (BBL Becton Dickinson) and tested for oxidase. Only oxidase-positive colonies were further identified using tDNA-PCR [14].

\section{Genotyping}

For each patient, all $P$. aeruginosa isolates exhibiting different colonial morphology on McConkey were first genotyped using alkaline cell lysis for DNA extraction and the random amplified polymorphic DNA fingerprinting technique with Ready-to-Go beads (Amersham Biosciences AB, Uppsala, Sweden) and primer ERIC2 (AAGTAAGTGACTGGGGTG AGCG) at an annealing temperature of $35^{\circ} \mathrm{C}$, as described previously [8]. This approach is designated ERIC2-PCR in the present study. This allowed a reduction in the number of isolates that were subsequently genotyped by the more laborious fluorescent amplified fragment length polymorphism analysis technique [7], since only single representatives of each ERIC2-PCR type were further genotyped by this procedure.

\section{RESULTS}

Table 1 presents the epidemiological and sampling data of the patients included.

Genotyping of P. aeruginosa was performed for 50 patients (26 male, sex not recorded for two; median age (range) 7 yrs and 11 months (9 months-31 yrs and 4 months)), including one sibling pair. A total of 427 environmental samples were obtained and genotyped with a median number of eight samples per patient (mean (range) samples 8.5 (4-23)). Nurses were asked to collect a minimum of four samples per home, with at least one sample from the kitchen, bathroom, toilet and the patient's nebuliser. Complete sampling was carried out for 39 out of 50 patients. Overall, one-third and one-fifth of the samples originated from the bathroom and the kitchen, respectively (table 2), for which $70.8 \%$ originated from the drains. The environmental samples were taken within 1 month of the positive sputum culture for $P$. aeruginosa in 37 of the 50 patients and within 1 week for 29 of these 37 patients (table 1). Environmental samples were also taken and processed for three patients for whom isolation of $P$. aeruginosa by the referring centre could not be confirmed by the reference laboratory. For one of these patients, an identical $P$. aeruginosa genotype was encountered in the sink drain 6 months before the first confirmed isolation of $P$. aeruginosa in the patient. A total of 25 environmental samples from 17 houses harboured $P$. aeruginosa. This corresponded to 18 patients, including one sibling pair. The median number of samples taken from houses with $P$. aeruginosa (nine samples per house) was not statistically different from the median number taken from all houses (eight samples per house) and the locations from which the samples were taken (i.e. bathroom kitchen, drain or tap) did not differ globally either. The vast majority of $P$. aeruginosapositive samples were recovered from the bathroom (i.e. 18 out of $25,72 \%$ ). For 12 out of the 25 patients for which $P$. aeruginosa could be recovered from the house, the house isolate was different from the patient isolate. These 12 samples came from nine patients (including one sibling pair) at eight different houses. A total of 13 of 25 environmental samples harboured $P$. aeruginosa with an identical genotype to that of the patient. The 13 samples came from nine patients at nine different houses. $P$. aeruginosa was found in only one of the 75 samples taken from the 48 nebulisers of the 50 patients. The latter $P$. aeruginosa isolate had an identical genotype to that of the patient. Another six nebulisers from six different patients harboured other bacteria, with one nebuliser contaminated with Stenotrophomonas maltophilia. The S. maltophilia genotype was different from the genotypes of the two positive samples for S. maltophilia found in the home environment of this patient. However, this patient had no positive sputum culture for S. maltophilia. The other bacteria cultured from the nebulisers are not usually considered to be of pathological significance for CF patients. It should be noted that in the present study, the nebuliser samples were only processed to recover gram-negative organisms. $P$. aeruginosa could not be recovered from any of the three private swimming pools of the presently studied CF patients. The sibling pair in the study (patients 18 and 21) was apparently simultaneously newly infected by the same strain of $P$. aeruginosa. The source of infection could not be identified, as the environmental $P$. aeruginosa recovered had a different genotype. By consulting the previously published national Belgian database of $P$. aeruginosa genotypes [7], the $P$. aeruginosa genotypes of the newly infected patients were also compared with the genotypes of $P$. aeruginosa cultured from chronically infected $\mathrm{CF}$ patients followed at the same centre. In three patients, followed at two different centres, the genotypes of the newly acquired $P$. aeruginosa strain were identical to the genotype of already chronically infected patients from their own centres. 


\begin{tabular}{|c|c|c|c|c|}
\hline \multirow[t]{2}{*}{ Sampling site } & \multirow[t]{2}{*}{ Samples } & \multicolumn{3}{|c|}{ Positive samples } \\
\hline & & Identical $^{\#}$ & Different & $\%$ \\
\hline Bathroom & $146(34.2)$ & 8 & 10 & 12.4 \\
\hline Bath drain & 39 & 3 & 4 & 17.9 \\
\hline Bath showerhead & 2 & 0 & 0 & 0 \\
\hline Bath tap & 7 & 1 & 0 & 14.3 \\
\hline Shower drain & 17 & 1 & 2 & 17.6 \\
\hline Shower tap & 3 & 0 & 0 & 0 \\
\hline Showerhead & 8 & 0 & 0 & 0 \\
\hline Toilet & 5 & 0 & 1 & 20.0 \\
\hline Toothbrush & 1 & 0 & 0 & 0 \\
\hline Washbasin drain & 49 & 3 & 2 & 10.2 \\
\hline Washbasin tap & 15 & 0 & 1 & 6.7 \\
\hline Kitchen & $87(20.4)$ & 2 & 2 & 4.9 \\
\hline Sink drain & 60 & 2 & 1 & 5.0 \\
\hline Sink tap & 22 & 0 & 1 & 4.5 \\
\hline Other & 5 & 0 & 0 & 0 \\
\hline Toilet room & $68(15.9)$ & 2 & 0 & 2.9 \\
\hline Toilet & 56 & 2 & 0 & 3.6 \\
\hline Washbasin drain & 11 & 0 & 0 & 0 \\
\hline Washbasin tap & 1 & 0 & 0 & 0 \\
\hline Nebuliser & 75 (17.6) & 1 & 0 & 13.3 \\
\hline Diverse & $51(11.9)$ & 0 & 0 & 0 \\
\hline Animal & 1 & 0 & 0 & 0 \\
\hline Aquarium & 7 & 0 & 0 & 0 \\
\hline Bedroom & 3 & 0 & 0 & 0 \\
\hline Plant & 3 & 0 & 0 & 0 \\
\hline Swimming pool & 3 & 0 & 0 & 0 \\
\hline Tumble dryer & 2 & 0 & 0 & 0 \\
\hline Washing machine & 3 & 0 & 0 & 0 \\
\hline Washroom & 6 & 0 & 0 & 0 \\
\hline Other inside house & 19 & 0 & 0 & 0 \\
\hline Other outside house & 4 & 0 & 0 & 0 \\
\hline Total & 427 & 13 & 12 & 5.9 \\
\hline
\end{tabular}

Data are presented as $\mathrm{n}$ or $\mathrm{n}(\%)$, unless otherwise indicated. ${ }^{\#}$ : genotype of the environmental isolate identical to the genotype from the patient genotype; $\because$ genotype of the environmental isolate different to the genotype from the patient genotype

\section{DISCUSSION}

$P$. aeruginosa is the most important pathogen in patients with CF. The origin of $P$. aeruginosa infection in patients with CF has not yet been clearly established, and the role of the environment as a source of $P$. aeruginosa acquisition in CF patients is still difficult to ascertain and remains a matter of debate [11, 12]. Patients with CF rarely appear to share genotypes, unless they are siblings or close friends, suggesting that patient-topatient transmission is rare and requires intense and longstanding contact. P. aeruginosa is a typical water organism and has been isolated from a number of environmental reservoirs $[9,10]$. The wide distribution of $P$. aeruginosa genotypes established in young $\mathrm{CF}$ children suggests acquisition from environmental reservoirs [15]. $P$. aeruginosa has been recovered from environmental sources in both in- and outpatient healthcare settings, e.g. sinks, tap water in a paediatric ward [16], toys, baths, hand soaps [17], pulmonary function equipment, hospital drains [18], whirlpool baths [19], dental equipment [20], and the hands of healthcare workers and CF patients [16-18]. The possibility of environment-to-patient transmission of these strains has been studied by DORING et al. [16], who described the possibility of environment-to-patient transmission of P. aeruginosa in a hospital setting. Doring et al. [16] suggested that the ability of CF sputum to facilitate survival of $P$. aeruginosa may be an important issue for strain transmission. During a cross-infection outbreak, JONES et al. [21] looked for the presence of epidemic strains in the environment of a CF centre and concluded that aerosol dissemination may be the most important factor in patient-to-patient spread of epidemic strains of $P$. aeruginosa.

In contrast to the extensive studies on the presence of $P$. aeruginosa in the hospital environment [16-20, 22-24] and on patient-to-patient transmission, there are only a few reports on the prevalence of $P$. aeruginosa in the home environment of both CF and non-CF patients (table 3) [22, 25-28]. These studies provide conflicting results. None of them studied the possibility of environment-to-patient transmission, neither did they compare genotypes of $P$. aeruginosa strains recovered from either patients or from their home environment.

To the best of the present authors' knowledge, the current study is the first to look for the presence of $P$. aeruginosa in the home environment of newly $P$. aeruginosa-infected CF patients and to compare genotypes of the environmental strains with the genotypes found in the patients.

In the present study, P. aeruginosa could be cultured from $5.9 \%$ of the home environmental samples in $34 \%$ of the houses. The data are comparable to data found in non-CF houses, although comparison is difficult because of different methodologies; however, a lower environmental contamination with $P$. aeruginosa is observed in CF houses compared with the other studies. The different findings might be explained by the following differences in procedures: 1) different sampling sites were tested, i.e. the present study did not investigate vegetables, which were an important source of $P$. aeruginosa in the study of MORTENSEN et al. [27]; 2) a different sampling methodology was used, i.e. $100-\mathrm{mL}$ aliquots of tap water were collected in the study by BARBEN et al. [26] versus 5-mL aliquots in the present study and cultures of standing water before the taps were used in the mornings in the studies of BARBEN et al. [26] and REGNATH et al. [25]; 3) different cleaning procedures were carried out by the patients; and 4) perhaps most importantly, the different colonisation status of the CF patients investigated, i.e. noncolonised patients in the present study, and $P$. aeruginosa-colonised patients in the other studies. However, the colonisation status is not always explicitly described in all other studies.

Although previous studies occasionally detected strains with the same (geno)type in the healthcare environment and in patients [16, 17, 24, 29], it remains unclear whether patients were the initial source of environmental contamination or whether the strains from the contaminated environmental source infected the patients. In the present study, for nine of the patients, the genotype of the environmental $P$. aeruginosa 
TABLE 3 Studies on the prevalence of Pseudomonas aeruginosa in cystic fibrosis (CF) and non-CF houses

\begin{tabular}{l}
$\begin{array}{l}\text { First author } \\
\text { [ref.] }\end{array}$ \\
\hline
\end{tabular}

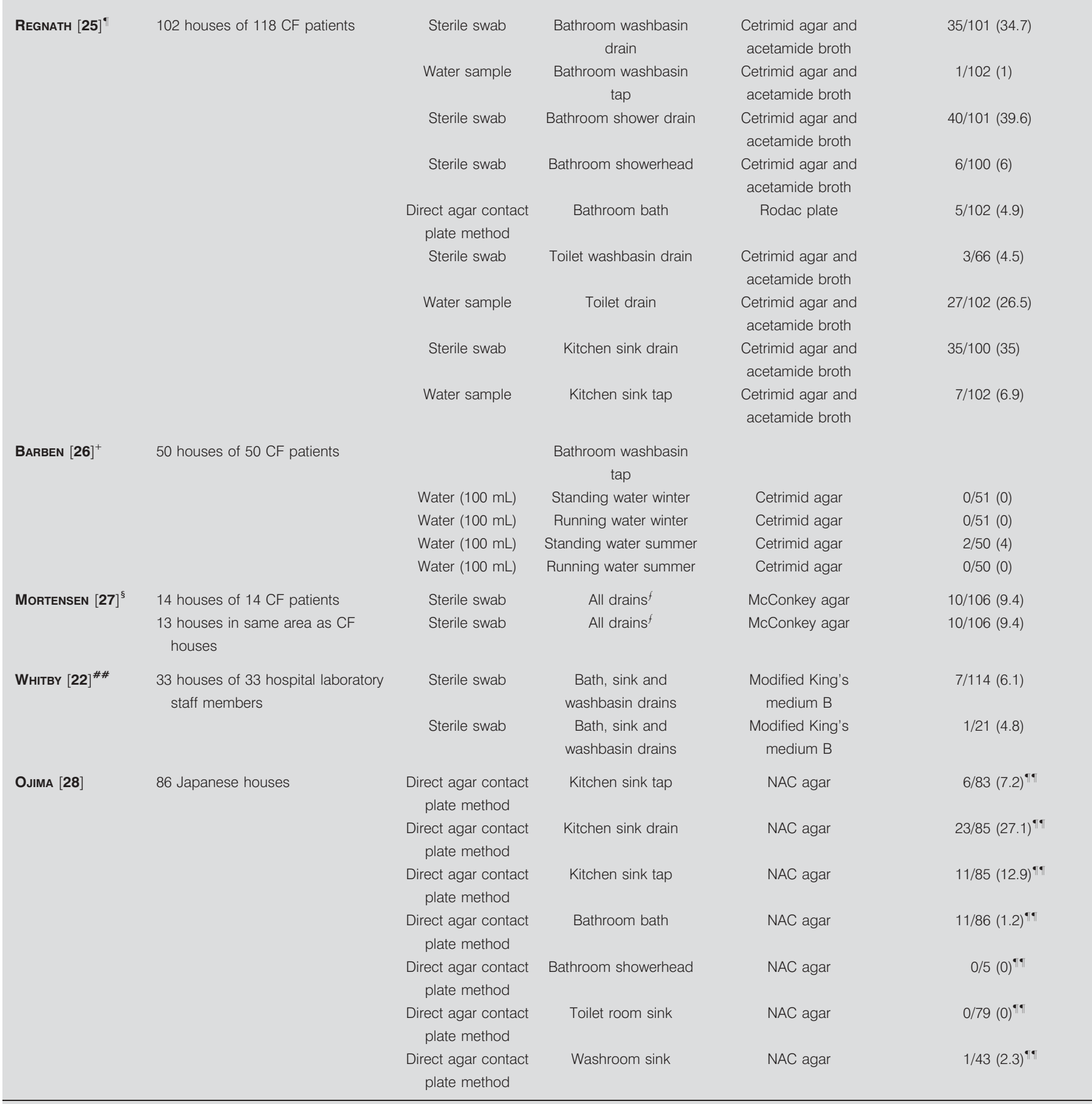

\#: acetamide broth (Fluka AG, Buchs, Switzerland); cetrimid agar, Rodac plate, McConkey agar and King's medium B (Becton Dickinson, Franklin Lakes, NJ, USA); and nalidixic acid cetrimide (NAC) agar (Nissui Pharmaceuticals, Tokyo, Japan). ': P. aeruginosa colonisation status of patients known for 88 patients, of whom 28 were colonised. Age ranged from 7 months to $41 \mathrm{yrs}$ (mean $11 \mathrm{yrs).} P$. aeruginosa was detected in $71.6 \%$ of the CF-houses. ${ }^{+}$: ages and colonisation status for $P$. aeruginosa of patients unknown. ${ }^{\S}$ : patients colonised with Burkholderia cepacia. Ages and colonisation status for $P$. aeruginosa of patients unknown. ${ }^{f}$ : all drains $=$ kitchen sink drains $+^{+}$ shower heads + refrigerator drain pan + bathtub drains. ${ }^{\# \#}: P$. aeruginosa detected in four out of 33 houses. " : number of houses with $P$. aeruginosa in the specified location/total number of houses examined for this location (percentages of households in which $P$. aeruginosa is detected). 
isolate was identical to that of $P$. aeruginosa isolated from the patient's sputum or nasopharyngeal aspirate. Therefore, an environmental strain could have been a possible source of infection for only nine of $50(18 \%)$ of the newly $P$. aeruginosainfected CF patients. As regular prospective home environmental cultures before the first positive $P$. aeruginosa infection in the patient are lacking from the present study, the authors cannot definitely conclude that the environmental strains were the source of infection or conversely that contamination of the environment was caused by the patient. However, the present study indicates that there is no overwhelming $P$. aeruginosa contamination of the home environment of newly infected CF patients. As the vast majority of environmental $P$. aeruginosa isolates were found in the bathroom of the patients, it would be advisable to pay special attention to the cleaning and disinfecting procedures for bathrooms. Compared with data from other studies [30,31], contamination of the nebulisers with $P$. aeruginosa in the present study was low. These findings suggest that the specific disinfecting procedures of the nebuliser equipment, as advised by the medical committee of the Belgian CF patient association [32], are effective.

In only three $(6 \%)$ of the 50 newly infected patients could there have been patient-to-patient transmission within the same CF centre. As far as was traceable, this transmission remains unexplained, as there was no close relationship between the patients, they were seen in segregated consultations, they were never hospitalised at the same time and they never attended the same CF rehabilitation centre or CF summer camp. None of these genotypes belonged to a cluster of genotypes established previously for Belgian colonised CF patients [7]. These results confirm the low patient-to-patient transmission percentages after the introduction of segregation measures in the $\mathrm{CF}$ centres [33].

Since the present study results suggest that neither home environment nor other patients are important sources of $P$. aeruginosa infection in CF patients, the question remains regarding the actual source of an initial infection. Apart from wet surfaces, the potential sources of $P$. aeruginosa in the home environment mentioned in literature are washing cloths, sponges for cleaning [28] and vegetables [27, 34]. As $P$. aeruginosa is widespread in soil and water, acquisition from the environment outside the house is probable. More studies are needed to elucidate the role of these potential infecting sources of $P$. aeruginosa in CF patients.

In conclusion, to the best of the present authors' knowledge, the current study is the first to look for the presence of Pseudomonas aeruginosa in the home environment of cystic fibrosis patients newly infected with Pseudomonas aeruginosa and to compare genotypes of environmental Pseudomonas aeruginosa with the genotypes of the strains recovered from these patients. Low percentages of possible home environment-to-patient transmission $(18 \%)$ and of patient-to-patient transmission within the same CF centre $(6 \%)$ are reported. In addition, the present study clearly shows that there is no overwhelming contamination level with Pseudomonas aeruginosa in the home environment of this population of newly Pseudomonas aeruginosa-infected patients. Contamination seems to occur mainly in the bathroom of the patient, suggesting that this place should be targeted for more rigorous cleaning procedures. More studies are needed to elucidate the role of other potential infecting sources of Pseudomonas aeruginosa in cystic fibrosis patients.

\section{ACKNOWLEDGEMENTS}

The authors wish to thank the following cystic fibrosis nurses for their help in this study: M. Vanderkerken, A. Raman, L. Boulanger, K. Colpaert, C. Dutranoix, V. Hubaux, A. Baudelot, F. de la Colette, Y. Jamoye, C. Vandekerckhove and H. Felix.

\section{REFERENCES}

1 Moskwa P, Lorentzen D, Excoffon KJ, et al. A novel host defense system of airways is defective in cystic fibrosis. Am J Respir Crit Care Med 2007; 175: 174-183.

2 Boucher RC. Airway surface dehydration in cystic fibrosis: pathogenesis and therapy. Annu Rev Med 2007; 58: 157-170.

3 Kosorok MR, Zeng L, West SE, et al. Acceleration of lung disease in children with cystic fibrosis after Pseudomonas aeruginosa acquisition. Pediatr Pulmonol 2001; 32: 277-287.

4 Nixon GM, Armstrong DS, Carzino R, et al. Clinical outcome after early Pseudomonas aeruginosa infection in cystic fibrosis. J Pediatr 2001; 138: 699-704.

5 Grothues D, Koopmann U, von der Hardt H, Tummler B. Genome fingerprinting of Pseudomonas aeruginosa indicates colonization of cystic fibrosis siblings with closely related strains. J Clin Microbiol 1988; 26: 1973-1977.

6 Ojeniyi B, Frederiksen B, Hoiby N. Pseudomonas aeruginosa cross-infection among patients with cystic fibrosis during a winter camp. Pediatr Pulmonol 2000; 29: 177-181.

7 Van Daele S, Vaneechoutte M, De Boeck K, et al. Survey of Pseudomonas aeruginosa genotypes in colonised cystic fibrosis patients. Eur Respir J 2006; 28: 740-747.

8 Van Daele SG, Franckx H, Verhelst R, et al. Epidemiology of Pseudomonas aeruginosa in a cystic fibrosis rehabilitation centre. Eur Respir J 2005; 25: 474-481.

9 Pirnay JP, Matthijs S, Colak H, et al. Global Pseudomonas aeruginosa biodiversity as reflected in a Belgian river. Environ Microbiol 2005; 7: 969-980.

10 Green SK, Schroth MN, Cho JJ, Kominos SK, Vitanzajack VB. Agricultural plants and soil as a reservoir for Pseudomonas aeruginosa. Appl Microbiol 1974; 28: 987-991.

11 Steinkamp G, Ullrich G. Different opinions of physicians on the importance of measures to prevent acquisition of Pseudomonas aeruginosa from the environment. J Cyst Fibros 2003; 2: 199-205.

12 Ullrich G, Wiedau-Gors S, Steinkamp G, Bartig HJ, Schulz W, Freihorst J. Parental fears of Pseudomonas infection and measures to prevent its acquisition. J Cyst Fibros 2002; 1: $122-130$.

13 Doring G, Conway SP, Heijerman HG, et al. Antibiotic therapy against Pseudomonas aeruginosa in cystic fibrosis: a European consensus. Eur Respir J 2000; 16: 749-767.

14 Baele M, Baele P, Vaneechoutte M, et al. Application of tRNA intergenic spacer PCR for identification of Enterococcus species. J Clin Microbiol 2000; 38: 4201-4207.

15 Burns JL, Gibson RL, McNamara S, et al. Longitudinal assessment of Pseudomonas aeruginosa in young children with cystic fibrosis. J Infect Dis 2001; 183: 444-452.

16 Doring G, Jansen S, Noll H, et al. Distribution and transmission of Pseudomonas aeruginosa and Burkholderia 
cepacia in a hospital ward. Pediatr Pulmonol 1996; 21: 90-100.

17 Zimakoff J, Hoiby N, Rosendal K, Guilbert JP. Epidemiology of Pseudomonas aeruginosa infection and the role of contamination of the environment in a cystic fibrosis clinic. J Hosp Infect 1983; 4: 31-40.

18 Speert DP, Campbell ME. Hospital epidemiology of Pseudomonas aeruginosa from patients with cystic fibrosis. J Hosp Infect 1987; 9: 11-21.

19 Berrouane YF, McNutt LA, Buschelman BJ, et al. Outbreak of severe Pseudomonas aeruginosa infections caused by a contaminated drain in a whirlpool bathtub. Clin Infect Dis 2000; 31: 1331-1337.

20 Jensen ET, Giwercman B, Ojeniyi B, et al. Epidemiology of Pseudomonas aeruginosa in cystic fibrosis and the possible role of contamination by dental equipment. J Hosp Infect 1997; 36: 117-122.

21 Jones AM, Govan JR, Doherty CJ, et al. Identification of airborne dissemination of epidemic multiresistant strains of Pseudomonas aeruginosa at a CF centre during a cross infection outbreak. Thorax 2003; 58: 525-527.

22 Whitby JL, Rampling A. Pseudomonas aeruginosa contamination in domestic and hospital environments. Lancet 1972; 1: 15-17.

23 Romling U, Wingender J, Muller H, Tummler B. A major Pseudomonas aeruginosa clone common to patients and aquatic habitats. Appl Environ Microbiol 1994; 60: 1734-1738.

24 Bosshammer J, Fiedler B, Gudowius P, von der Hardt H, Romling U, Tummler B. Comparative hygienic surveillance of contamination with pseudomonads in a cystic fibrosis ward over a 4-year period. J Hosp Infect 1995; 31: 261-274.

25 Regnath T, Kreutzberger M, Illing S, Oehme R, Liesenfeld O. Prevalence of Pseudomonas aeruginosa in households of patients with cystic fibrosis. Int J Hyg Environ Health 2004 207: 585-588.

26 Barben J, Hafen G, Schmid J. Pseudomonas aeruginosa in public swimming pools and bathroom water of patients with cystic fibrosis. J Cyst Fibros 2005; 4: 227-231.

27 Mortensen JE, Fisher MC, LiPuma JJ. Recovery of Pseudomonas cepacia and other Pseudomonas species from the environment. Infect Control Hosp Epidemiol 1995; 16: 30-32.

28 Ojima M, Toshima Y, Koya E, et al. Hygiene measures considering actual distributions of microorganisms in Japanese households. J Appl Microbiol 2002; 93: 800-809.

29 Wolz C, Kiosz G, Ogle JW, et al. Pseudomonas aeruginosa cross-colonization and persistence in patients with cystic fibrosis. Use of a DNA probe. Epidemiol Infect 1989; 102: 205-214.

30 Pitchford KC, Corey M, Highsmith AK, et al. Pseudomonas species contamination of cystic fibrosis patients' home inhalation equipment. J Pediatr 1987; 111: 212-216.

31 Hutchinson GR, Parker S, Pryor JA, et al. Home-use nebulizers: a potential primary source of Burkholderia cepacia and other colistin-resistant, gram-negative bacteria in patients with cystic fibrosis. J Clin Microbiol 1996; 34 584-587.

32 Reychler G, Aarab K, Van Ossel C, et al. In vitro evaluation of efficacy of 5 methods of disinfection on mouthpieces and facemasks contaminated by strains of cystic fibrosis patients. J Cyst Fibros 2005; 4: 183-187.

33 Frederiksen B, Koch C, Hoiby N. Changing epidemiology of Pseudomonas aeruginosa infection in Danish cystic fibrosis patients (1974-1995). Pediatr Pulmonol 1999; 28: 159-166.

34 Wright C, Kominos SD, Yee RB. Enterobacteriaceae and Pseudomonas aeruginosa recovered from vegetable salads. Appl Environ Microbiol 1976; 31: 453-454. 